\title{
Contribution of biodiversity to ecosystem functioning: a non-equilibrium thermodynamic perspective
}

\author{
Amit CHAKRABORTY ${ }^{1,2}$, B Larry LI ${ }^{1,2^{*}}$ \\ ${ }^{1}$ Ecological Complexity and Modeling Laboratory, Department of Botany and Plant Sciences, University of California, Riverside \\ CA 92521-0124, USA; \\ ${ }^{2}$ XIEG-UCR International Center for Arid Land Ecology, University of California, Riverside CA 92521, USA
}

\begin{abstract}
Ecosystem stays far from thermodynamic equilibrium. Through the interactions among biotic and abiotic components, and encompassing physical environments, ecosystem forms a dissipative structure that allows it to dissipate energy continuously and thereby remains functional over time. Biotic regulation of energy and material fluxes in and out of the ecosystem allows it to maintain a homeostatic state which corresponds to a self-organized state emerged in a non-equilibrium thermodynamic system. While the associated self-organizational processes approach to homeostatic state, entropy (a measure of irreversibility) degrades and dissipation of energy increases. We propose here that at a homeostatic state of ecosystem, biodiversity which includes both phenotypic and functional diversity, attains optimal values. As long as biodiversity remains within its optimal range, the corresponding homeostatic state is maintained. However, while embedded environmental conditions fluctuate along the gradient of accelerating changes, phenotypic diversity and functional diversity contribute inversely to the associated self-organizing processes. Furthermore, an increase or decrease in biodiversity outside of its optimal range makes the ecosystem vulnerable to transition into a different state.
\end{abstract}

Keywords: ecosystem; self-organization; non-equilibrium thermodynamics; functional diversity; phenotypic diversity

Every living organism has a life cycle comprising several life processes such as birth, death, growth, reproduction, energy consumption, and energy conversion. These life processes have effects on, and responded to, the local biotic and abiotic environment in which an organism lives in and thrives on. Such intrinsic abilities engage multiple organisms into a network of interactions in which the extent of the effects and responses of each participant vary simultaneously with other participants and are not necessarily symmetric in nature (Dunne et al., 2002; Proulx et al., 2005; Bluthgen et al., 2008). In that interaction network, the way organism functions being an antagonist, facilitator or mutualist represents its 'functional niche' - the ecological role that exerts for continuing survival and reproduction. Organisms with certain similarities among their functional niches (e.g., similar resource consumption rate) form a 'functional group'. Although members of a functional group apparently embrace similar ecological function, they differ in magnitude of effect and respond that they exert toward others and surrounding physical environments. Phe- notypic diversity of functionally equivalent organisms arises from individual's differential abilities to deal with other interacting members and variability in environmental conditions in which they interact. Phenotypic plasticity is one such inherent ability of organism that allows it to cope with environmental challenges often posed by the variation in consumer pressure, disturbances, abiotic environment, and predation risks (Agrawal, 2001). On the other hand, specializations particularly for resource acquisition, utilization and conservation allow organism to deal with competition from other members of the interaction network (Bolnick et al., 2003; Svanback et al., 2007). While multiple phenotypes compete with each other for resources, they undergo natural selection. In a relatively constant or low-stress environment, specialization is often favored over plasticity, allowing utilization of resources more efficiently (Callaway et al., 2003; Chakraborty and $\mathrm{Li}, 2009)$. Such specializations allow population

Received 2010-10-18, accepted 2010-10-26

doi: 10.3724/SP.J.1227.2011.00071

* Corresponding author: BaiLian LI (E-mail: bai-lian.li@ucr.edu) 
with specialized phenotypes to recover from low abundance in presence of competitors. On the other hand, plasticity is favored over specialization when the environment is variable and environmental cues are reliable so that individual can express the appropriate phenotype in the extant environment, and if there are higher associated costs for specialized phenotypes (Futuyma and Moreno, 1988). Such divergent roles of natural selection driven essentially by the extant environmental conditions are famously noted by Dobzhansky (1950); he postulated that natural selection in the tropic operates in a fundamentally different way than it does in temperate zones. Latter, this idea is formalized in general terms by MacArthur and Wilson (1967), named as the r-K selection continuum, an ideal ecologic vacuum. The r-endpoint of the continuum represents a quantitative extreme with no density effects and no competition, whereas the K-endpoint represents another extreme with highest density effects and strong competition. As the ecologic vacuum filled with populations, natural selection favor those that allocate most resources into maintenance and produce a few but extremely fit offspring (K-strategy) over those that allocate most resources into reproduction and produce as many total progeny as possible (r-strategy) (Pianka, 1970).

Multiple functional groups and encompassing physical environments all together form a complex interacting system called as 'ecosystems' (Pimm, 1984; Levin, 1998). The amount of energy and materials flowed into and dissipated from an ecosystem are determined by the interacting functional groups. Thus, the ecosystem properties such as productivity depend on energy fluxes and its retention and rate regulation (Odum, 1968). While each functional group simply operates for capturing, retaining, and converting energy and resources, their combined actions tend to have counterbalancing effects on environmental changes (Amaral et al., 1998; Kirchner and Weil, 2000; Norberg et al., 2001). These understandings are encapsulated into the concept of 'ecosystem homeostasis' which postulates that biotic regulation allows an ecosystem to maintain a stable state (Trojan, 1984), which is similar to much broadly defined metabolic optimum across all of life's major kingdoms (Makarieva et al., 2008). At a homeostatic state, biotic components and embedded physical environments remain within a range of numerical bounds. If one functional group fails to operate properly with the loss of many phenotypes, phenotypic diversity of other functional groups are enhanced to compensate that incurred damage, allowing ecosystems to maintain the homeostatic state (Ernest and Brown, 2001). When ecosystem experiences a severe outside disturbance, it may cause the biotic and abiotic components to alter the requisite numerical bounds for the extant homeostatic state, leading to shift into other homeostatic state over the course of time. Such self-organizing characteristics of ecosystems have often been recognized as undirected emergent properties (Kay et al., 1999; Li, 2000a, b, 2002). This view of ecosystem is in sheer contrast with the 'organismal ecosystem model' (Clements, 1916; Reiners, 1986). In the organismal model, ecosystem is viewed as a single living entity which evolves toward 'climax', where the system will be saturated with organisms, and the energy and resource used will be maximized. Thus, the emergence and surprises are common in this model as many organisms come in and go out over the course of evolution, and the evolution progresses as existing organisms alter encompassing physical environment making it hospitable for incoming organisms.

While viewing ecosystem as a complex interacting system, the second law of thermodynamics provides a physical basis for understanding and exploring self-organizing characteristics of ecosystems (Ulanowicz, 1972; Schneider and Kay, 1994; Li, 2000b, 2002; Jorgensen and Fath, 2004). According to the second law, any process involving energy fluxes through the interaction of multiple entities is directed towards a certain death, called as thermodynamic equilibrium, where entropy, a measure of irreversibility, should be maximized and the energy dissipation will completely be terminated. Biotic components of ecosystem, through interactions among themselves and with physical environments, continuously dissipate energy, keeping the ecosystem away from the thermodynamic equilibrium. Ecosystem, therefore, remains functional or operational by exchanging energy and materials with the outside environments and forming a dissipative structure that allows it to embrace continuous dissipation of energy. Thermodynamics of non-equilibrium systems insist that while a system stays away from thermodynamic equilibrium, an organizational steady state will emerge at which the system to be organized in a way that reduces or degrades entropy 
(Kestin, 1968; Helbing and Vicsek, 1999; Kay, 2000). Consequently, if environmental conditions permit, self-organization processes allow a system to build-up organizational structure that reduces entropy more effectively than if the dynamic and kinetic pathways for those structures were not available (Kay, 2000). Thus, it implies that ecosystem can exhibit self-organizational structures that correspond to there homeostatic states; as long as biotic components and physical environments remain within a range of numerical bounds, the homeostatic state will be maintained by associated self-organization processes.

Following the recent work of Aleshchenko and Bukvareva (2010), we propose here that the biodiversity, both phenotypic and functional diversity, attain a unique optimal value (D*) at a homeostatic state of ecosystem. As long as the biodiversity remains close to its optimal value (i.e., within an optimal range), the homeostatic state will be maintained (Fig. 1). This means that at $\mathrm{D}^{*}$ level, entropy and dissipation of energy will be optimized. Within the optimal range, if the biodiversity holds a numerical value below the $\mathrm{D}^{*}$ level, it reflects a lower level of energy dissipation than the required level at the homeostatic state. So, self-organization processes will be initiated through the enhancement of biodiversity, which will lead the system toward achieving the required level of energy dissipation for the optimized state. On the other hand, if biodiversity resides near but above the $\mathrm{D}^{*}$ level, it reflects relatively a higher entropy than its required level; so the self-organization processes will be initiated by lowering biodiversity level which will eventu- ally be resulted in a reduced, optimized entropy level.

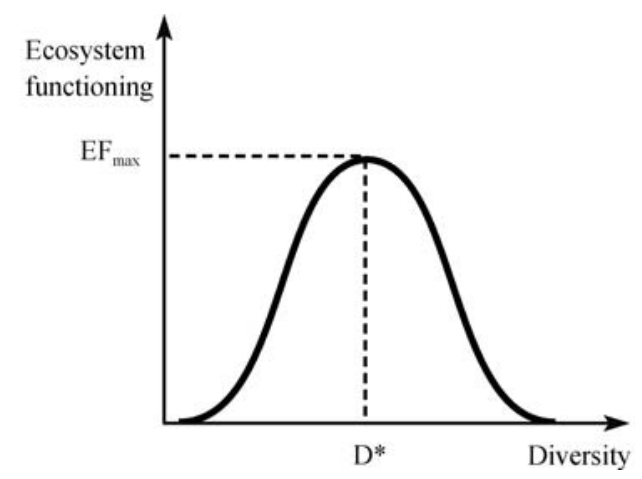

Fig. 1 Optimal biodiversity $\left(D^{\star}\right)$ corresponds to a homeostatic state of ecosystem where entropy and dissipation of energy are optimized. The homeostatic state, a self-organized state of ecosystem, is maintained as long as biodiversity remains within its optimal range.

While surrounding environment fluctuates over a spatiotemporal scale, functional diversity and phenotypic diversity contribute differently to self-organization processes. Along the gradient of accelerating environmental changes, phenotypic diversity increases to withstand increasing environmental variability, whereas functional diversity decreases due to increased complementarity effects resulting from reduced competitive interactions (Fig. 2) (Loreau, 1998). Moreover, an increase or decrease in biodiversity outside of its optimal range makes the system vulnerable to transition into a different state.

This particular view parallels to the recent idea of 'optimal biodiversity' presented by Aleshchenko and Bukvareva (2010), they illustrated this phenomenon

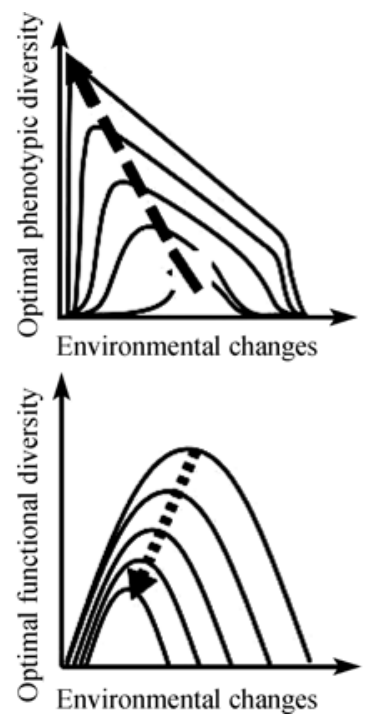

Fig. 2 Along the gradient of accelerating environmental changes, functional diversity decreases with increasing phenotypic diversity within the range of optimal biodiversity. 
using a hierarchical model connecting phenotypic diversity at the level of population into species diversity at a level of community.

\section{Acknowledgements}

This research was partially supported by the U.S. National Sci-

\section{References}

Agrawal A A. 2001. Phenotypic plasticity in the interactions and evolution of species. Science, 294(5541): 321-326.

Aleshchenko G M, Bukvareva E N. 2010. Two level hierarchical model of optimal biological diversity. Biology Bulletin, 37(1): 1-9.

Amaral L A, Buldyrev S V, Havlin S, et al. 1998. Power law scaling for a system of interacting units with complex internal structure. Physics Review Letters, 80(7): 1385-1388.

Bluthgen N, Frund J, Vazquez D P, et al. 2008. What do interaction network metrics tell us about specialization and biological traits. Ecology, 89(12): 3387-3399.

Bolnick D I, Svanback R, Fordyce J A, et al. 2003. The ecology of individuals: incidence and implications of individual specialization. The American Naturalist, 161(1): 1-28.

Callaway R M, Pennings S C, Richards C L. 2003. Phenotypic plasticity and interactions among plants. Ecology, 84(5): 1115-1128.

Chakraborty A, Li B L. 2009. Plant-to-plant direct competition for belowground resource in an overlapping depletion zone. Journal of Arid Land, 1(1): 9-15.

Clements F C. 1916. Plant Succession: An Analysis of the Development of Vegetation. Washington D. C.: Carnegie Institute.

Dobzhansky T. 1950. Evolution in tropics. American Scientist, 38: 209-221.

Dunne J A, Williams R J, Martinez N D. 2002. Food-web structure and network theory: the role of connectance and size. Proceedings of the National Academy of Sciences (USA), 99(20): 12917-12922.

Ernest S K M, Brown J H. 2001. Homeostasis and compensation: the role of species and resources in ecosystem stability. Ecology, 82(8): 2118-2132.

Futuyma D J, Moreno G. 1988. The evolution of ecological specialization. Annual Review of Ecology and Systematics, 19: 207-233.

Helbing D, Vicsek T. 1999. Optimal self-organization. New Journal of Physics, 1: 131-137.

Jorgensen S E, Fath B D. 2004. Application of thermodynamic principles in ecology. Ecological Complexity, 1(4): 267-280.

Kay J J, Regier H A, Boyle M, et al. 1999. An ecosystem approach for sustainability: addressing the challenge for complexity. Futures, 31(7): 721-742.

Kay J J. 2000. Ecosystems as self-organizing holarchic open systems: narratives and the second law of thermodynamics. In: Jorgensen S E, Muller F. Handbook of Ecosystem Theories and Management, Lewis publishers: CRC Press, 135-160.

Kestin J. 1968. A Course in Thermodynamics. New York: Hemisphere Press.

Kirchner J W, Weil A. 2000. Delayed biological recovery from extinc- ence Foundation's Biocomplexity Program (DEB-0421530) and Long-Term Ecological Research Program (Sevilleta LTER, DEB-0620482) and University of California Agricultural Experiment Station. Communication with Dr. Elena $\mathrm{N}$. BUKVAREVA at A. N. Severtsov Institute of Ecology and Evolution, Russian Academy of Sciences (Moscow), was very helpful to shape our view presented in this manuscript.

tions throughout the fossil record. Nature, 404: 177-180.

Levin S A. 1998. Ecosystems and the biosphere as complex adaptive systems. Ecosystems, 1(5): 431-436.

Li B L. 2000a. Fractal geometry applications in description and analysis of patterns and patch dynamics. Ecological Modeling, 132: 33-50.

Li B L. 2000b. Why is the holistic approach becoming so important in landscape ecology? Landscape and Urban Planning, 50: 27-41.

Li B L. 2002. A theoretical framework of ecological phase transitions for characterizing tree-grass dynamics. Acta Biotheoretica, 50(3): 141-154.

Loreau M. 1998. Biodiversity and ecosystem functioning: a mechanistic model. Proceedings of the National Academy of Sciences (USA), 95(10): 5632-5636

MacArthur R H, Wilson E O. 1967. The theory of island biogeography. Princeton: Princeton University Press, 203.

Makarieva A M, Gorshkov V G, Li B L, et al. 2008. Mean mass-specific metabolic rates are strikingly similar across life's major domains: Evidence for life's metabolic optimum. Proceedings of the National Academy of Sciences (USA), 105 (44): 16994-16999.

Norberg J, Swaney D P, Dushoff J, et al. 2001. Phenotypic diversity and ecosystem functioning in changing environments: a theoretical framework. Proceedings of the National Academy of Sciences (USA), 98(20): 11376-11381.

Odum E P. 1968. Energy flow in ecosystems: a historical review. American Zoologist, 8(1): 11-18.

Pianka E R. 1970. On r-and K selection. The American Naturalist, 104(940): 592-597.

Pimm S L. 1984. The complexity and stability of ecosystems. Nature, 307: 321-326.

Proulx R, Promislow D E L, Phillips P S. 2005. Network thinking in ecology and evolution. Trends in Ecology and Evolution, 20(6): 345-353.

Reiners W A. 1986. Complementary models for ecosystems. The American Naturalist, 127(1): 59-73.

Schneider E D, Kay J J. 1994. Life as a manifestation of the second law of thermodynamics. Mathematical and Computer Modeling, 19(6-8): 25-48.

Svanback R, Bolnick D I. 2007. Intraspecific competition drives increased resource use diversity within a natural population. Proceedings of the Royal Society London, Series B, 274 (1611): 839-844.

Trojan P. 1984. Ecosystem Homeostasis. Warszawa: Junk W and PWN-Polish Scientific Publishers,

Ulanowicz R E. 1972. Mass and energy flow in closed ecosystems. Journal of Theoretical Biology, 34(2): 239-253. 